TẠP CHÍ KHOA HỌC ĐÄI HỌC TÂN TRÀO
ISSN: 2354 - 1431
http://tckh.daihoctantrao.edu.vn/

\title{
HOẠT ĐộNG TƯ VẤN CỦA CỐ VẤN HỌC TẬP CHO SINH VIÊN CÁC TRƯờnG ĐẠI HỌC
}

\author{
Lý Văn Thành
}

Đại học Tân Trào

Mail: lykhaiphong2012@gmail.com

\section{Thông tin bài viết}

Ngày nhận bài:

$22 / 5 / 2020$

Ngày duyệt đăng:

20/9/2020

Từ khóa:

Cố vấn học tập, hoạt động

tu vấn, sinh viên, giảng

viên, truò̀ng đại học.

\section{Tóm tắt}

Tại các Trường Đại học ở Việt Nam, đào tạo theo hệ thống tín chỉ đã trở thành quy định bắt buộc của Bộ Giáo dục và Đào tạo, để hỗ trợ sinh viên có thể thực hiện quyền tự chủ của mình một cách hiệu quả nhất, một khâu quan trọng trong quy trình đào tạo theo hệ thống tín chỉ là bộ phận cố vấn học tập. Cố vấn học tập vừa đóng vai trò là giáo viên chủ nhiệm để quản lý $S V$, vừa đóng vai trò là chuyên gia tư vấn học tập, nghiên cứu khoa học và việc làm cho $S V$. Bài viết đề cập tới khái niệm, nội dung và các kỹ năng cần thiết để tư vấn cho $\mathrm{SV}$ của cố vấn học tập ở các trường đại học.

\section{1. Đặt vấn đề}

Cố vấn học tập (CVHT) - cụm từ được nhắc đến nhiều từ khi phương thức đào tạo tín chỉ được áp dụng ở bậc đào tạo đại học tại Việt Nam. Trước tiên phải khẳng định rằng, CVHT có vai trò đặc biệt quan trọng trong đào tạo theo tín chỉ và ảnh hưởng đến sự thành công trong học tập, rèn luyện của sinh viên (SV). Mỗi CVHT như là một "mắt xích" trong vòng tròn mối liên hệ giữa $\mathrm{SV}$ - chương trình đào tạo - nhà trường. Phần lớn các trường đại học và một số trường cao đẳng hiện nay đã có những văn bản quy định ghi rõ nhiệm vụ, vai trò, quyền lợi và trách nhiệm của CVHT. Tuy nhiên, kết quả thực hiện theo các văn bản cũng như nhiệm vụ và vai trò của CVHT ở mỗi trường lại rất khác nhau. Trong đào tạo theo tín chỉ, nhiệm vụ của CVHT là giúp cho quá trình cá nhân hóa học tập của $\mathrm{SV}$ được diễn ra một cách tốt nhất. Công việc của họ là tư vấn cho $\mathrm{SV}$ để các em tự tổ chức và kiểm soát tốt nhất tiến trình học tập của mình, giúp $\mathrm{SV}$ thực hiện được mục tiêu học tập. Bởi vậy, hoạt động tư vấn của CVHT có vai trò quan trọng đối với kết quả học tập và rèn luyện của $\mathrm{SV}$.

\section{Nội dung nghiên cứu}

\subsection{Khái niệm chung về cố vấn học tập}

Tại các Trường Đại học tại Việt Nam, đào tạo theo hệ thống tín chỉ đã trở thành quy định bắt buộc của Bộ Giáo dục và Đào tạo. Trong quá trình học, sinh viên phải chủ động trong việc lựa chọn ngành, chuyên ngành và môn học. Ngoài ra, sinh viên còn phải xây dựng kế hoạch học tập cho cả quá trình đào tạo cũng như từng năm học, kỳ học. Để hỗ trợ sinh viên có thể thực hiện quyền tự chủ của mình một cách hiệu quả nhất, một khâu quan trọng trong quy trình đào tạo theo hệ thống tín chỉ là bộ phận CVHT. Khi nghiên cứu về CVHT ở các trường đại học đã có một số công trình công bố các kết quả nghiên cứu cả về lý luận và thực tiễn, nhìn chung CVHT là người tư vấn hỗ trợ sinh viên phát huy tối đa khả năng học tập, rèn luyện và nghiên cứu khoa học, lựa chọn đăng ký học phần phù hợp để đáp ứng mục tiêu tốt nghiệp và khả năng tìm được việc làm sau khi ra trường, theo dõi quá trình học tập, rèn luyện của sinh viên nhằm giúp sinh viên điều chỉnh kịp thời hoặc đưa ra sự lựa chọn đúng trong 
quá trình học tập, đồng thời quản lý, hướng dẫn và chỉ đạo lớp được phân công phụ trách.

Qua quá trình tổng hợp các nghiên cứu theo chúng tôi: “CVHT là người quản lý và tư vấn học tập cho $\mathrm{SV}$ nhằm nâng cao hiệu quả, chất lượng đào tạo theo hệ thống tín chỉ ở các trường đại học".

CVHT có vai trò đặc biệt quan trọng, không thể thiếu trong đào tạo theo tín chỉ. CVHT vừa đóng vai trò là giáo viên chủ nhiệm để quản lý $\mathrm{SV}$, vừa đóng vai trò là chuyên gia tư vấn học tập, nghiên cứu khoa học và việc làm cho $\mathrm{SV}$. Với vai trò là người tư vấn, nhiệm vụ của CVHT gồm: Hỗ trợ SV tìm hiểu chương trình đào tạo, nắm rõ các quy định đào tạo theo tín chỉ, đặc biệt là quy trình đăng ký môn học và lập kế hoạch học tập cá nhân cho từng học kỳ và toàn khóa học; Hướng dẫn, tư vấn cho SV xây dựng kế hoạch học tập phù hợp với điều kiện của cá nhân và mục tiêu, yêu cầu của môn học; Hướng dẫn, tư vấn cho $\mathrm{SV}$ về phương pháp học tập, nghiên cứu khoa học và theo dõi việc học tập các học phần của SV; Hướng dẫn SV tiếp cận các nguồn học liệu; Phối hợp có hiệu quả với giảng viên dạy các học phần để nắm bắt kịp thời tình hình học tập của SV...[5, tr. 76-81].

\subsection{Hoạt động tu vấn học tập theo hệ thống tín chi của CVHT}

Để đảm nhiệm tốt nhiệm vụ của mình, CVHT cần có các hoạt động tư vấn hướng dẫn cho SV. Theo chúng tôi: "Hoạt động tư vấn học tập của CVHT là sụ vận dụng kiến thức, kinh nghiệm tu vấn học tập của CVHT vào việc xây dựng mối quan hệ cởi mở, chân thành, tin cậy và chuẩn mục với $S V$, trao đổi thông tin để cung cấp giải pháp, giúp SV giải quyết nhũng khó khăn mà ho gặp phải trong hoc tập, nghiên cứu khoa học, huoóng nghiệp... nhằm tích lũy đủ hệ thống môn hoc theo trình tụ quy dịnh của chuong trình đào tạo để đạt được văn bằng một cách tốt nhất”.

Hoạt động tư vấn học tập được hình thành trên cơ sở vận dụng hiểu biết, tri thức và giá trị nghề nghiệp. Đó là những hiểu biết về mục đích của tư vấn học tập, về nguyên tắc, giá trị của tư vấn học tập và các hiểu biết, kinh nghiệm trong giảng dạy và kinh nghiệm xã hội. Vì vậy, tiêu chí để lựa chọn CVHT ở các trường đại học phải là người nắm vững đường lối, chủ trương của Đảng, chính sách, pháp luật của Nhà nước; Hiểu biết và vận dụng tốt quy chế đào tạo và quy chế về công tác HS-SV của Bộ Giáo dục và Đào tạo, của trường (mục tiêu, chương trình đào tạo, các hình thức đào tạo, quy chế, quy trình đào tạo, quy định về chế độ chính sách và công tác HS-SV; Là GV có cùng chuyên ngành, am hiểu sâu chuyên môn; có kinh nghiệm giảng dạy, quản lý $\mathrm{SV}$ và am hiểu về chức năng CVHT; Có tinh thần trách nhiệm cao trong công tác... [4, tr. 259-261].

\subsection{Nội dung co bản về hoạt động tu vấn của CVHT ở các truờng đại học}

Nội dung cơ bản về hoạt động tư vấn học tập của CVHT là xây dựng được mối quan hệ tin cậy, chuẩn mực với $\mathrm{SV}$; trao đổi thông tin và đưa ra giải pháp giúp giải quyết những vấn đề mà các em đang gặp phải. Như vậy, quy trình để CVHT tư vấn cho SV sẽ bao gồm 3 giai đoạn [2]:

Giai đoạn 1: Xây dựng mối quan hệ cởi mở, chân thành, tin cậy và chuẩn mực với $\mathrm{SV}$ : Mối quan hệ giữa CVHT với $\mathrm{SV}$ là mối quan hệ công việc, được nhà trường thừa nhận, là mối quan hệ cởi mở, chân thành. Đây là điều kiện để SV sẵn sàng chia sẻ với CVHT về những khó khăn mà họ đang gặp phải, từ đó tự tin đưa ra cách thức giải quyết phù hợp; mối quan hệ giữa CVHT với $S V$ phải là mối quan hệ tin cậy. Niềm tin sẽ tạo cho SV tâm thế tích cực hợp tác, đón nhận lời khuyên của CVHT, đồng thời tạo ra tình cảm tốt đẹp, mối quan hệ lâu dài và gắn bó với CVHT (thông thường $\mathrm{CVHT}$ thường theo $\mathrm{SV}$ từ đầu khóa đến khi kết thúc ra trường). Đồng thời, mối quan hệ giữa CVHT với $\mathrm{SV}$ phải là mối quan hệ chuẩn mực. Đây không chỉ là cốt lõi tạo nên uy tín của CVHT, tạo niềm tin đối với $\mathrm{SV}$ mà còn là điều kiện đảm bảo sự tôn trọng, chấp hành nghiêm chỉnh nội quy, quy chế của nhà trường và của ngành Giáo dục.

Giai đoạn 2: Thu thập thông tin trong tư vấn học tập theo hệ thống tín chỉ (HTTC). Thu thập thông tin là một khâu quan trọng trong tư vấn học tập theo HTTC. CVHT sẽ đưa ra quyết định đúng đắn nhất nếu họ thu thập được những thông tin đầy đủ và chính xác về tình hình của $S V$. Vì vậy, CVHT cần thu thập thông tin để hiểu chính xác những vấn đề mà $\mathrm{SV}$ đang gặp phải trong học tập, nghiên cứu khoa học và hướng nghiệp; hiểu được nguyện vọng của $\mathrm{SV}$ và hiểu quy chế để đưa ra phương án chính xác giải quyết những vấn đề mà các em đang gặp phải. Để thu thập chính xác thông tin về tư vấn học tập, $G V$ cần có kỹ năng hỏi $\mathrm{SV}$, kỹ năng lắng nghe $\mathrm{SV}$, kỹ năng kiểm tra các nguồn thông tin, tài liệu. CVHT có thể kiểm tra các nguồn thông tin mà $\mathrm{SV}$ cung cấp qua phòng chức năng như: Phòng Đào tạo, Phòng Công tác HS-SV, qua khoa chuyên môn hay qua GV giảng dạy bộ môn. Đồng thời, CVHT phải nghiên cứu thật kỹ các văn bản pháp quy về đào tạo theo tín chỉ, niên giám của nhà 
trường,... như vậy mới có thể đưa ra phương án đúng đắn và hiệu quả nhất để giải quyết các vấn đề của $\mathrm{SV}$.

Giai đoạn 3: Tìm ra giải pháp giúp $\mathrm{SV}$ giải quyết những vấn đề mà họ đang gặp phải. Bởi trong tư vấn học tập theo HTTC không đơn giản là giải đáp thắc mắc, hướng dẫn giải quyết các vấn đề mà họ gặp phải mà còn giúp $\mathrm{SV}$ có khả năng tốt nhất để tự quyết định các vấn đề trong học tập, nghiên cứu khoa học, việc làm,...của $\mathrm{SV}$. Việc tìm ra giải pháp giúp $\mathrm{SV}$ hiểu được các quy định về đào tạo thắc mắc và tự đưa ra quyết định liên quan đến nội dung đó. Việc tìm ra giải pháp của CVHT phải đưa ra được đầy đủ thông tin và các quy định có tính chất pháp quy liên quan đến vấn đề của $\mathrm{SV}$; chỉ ra các hướng giải quyết và phân tích ưu, nhược điểm của các hướng đó để SV tự đưa ra quyết định. Để giúp $\mathrm{SV}$ tìm ra giải pháp có hiệu quả, $\mathrm{GV}$ cần biết mô tả, phân tích và đánh giá thông tin, sử dụng phương tiện ngôn ngữ và phi ngôn ngữ để diễn tả vấn đề.

\subsection{Một số kỹ năng co bản trong hoạt động tur vấn, hướng dẫn của CVHT}

Hoạt động của CVHT đòi hỏi phải có một số kỹ năng nhất định trong quá trình tư vấn, hướng dẫn, giúp đỡ SV. Để làm tốt nhiệm vụ này $\mathrm{CVHT}$ cần phải có một số kỹ năng cơ bản sau đây [3, tr. 176-177]:

\subsubsection{Kỹy Năng đặt câu hỏi}

Các nghiên cứu của một số tác giả cho rằng, trước khi phát triển một chương trình đào tạo, việc tư vấn cho người học là quan trọng nhất, phải có kỹ thuật tư vấn thì mới có hiệu quả, do đó, những người tư vấn cần có kỹ năng đặt câu hỏi và kỹ năng cung cấp thông tin tốt. Khi CVHT đặt câu hỏi cho $\mathrm{SV}$, cần để ý đến suy nghĩ, cảm xúc, thái độ của $\mathrm{SV}$ và chuẩn bị cẩn thận những câu hỏi dự định hỏi, bởi các câu hỏi có tác động rất mạnh đến tinh thần của các em nên cần thể hiện được sự khích lệ trong câu hỏi.

CVHT cần có kỹ năng đặt câu hỏi để hiểu vấn đề; có kỹ năng thu thập thông tin từ các nguồn; can thiệp vào tiến trình học tập để đáp ứng nhu cầu của $\mathrm{SV}$, các CVHT cần có kỹ năng tư vấn thể hiện ở việc trò chuyện được với $\mathrm{SV}$, hiểu được tâm lý của $\mathrm{SV}$ bao gồm năng lực, sở thích, điều kiện sinh hoạt, hoàn cảnh kinh tế,... để có thể hướng dẫn SV xây dựng kế hoạch học tập và có thể hỗ trợ $\mathrm{SV}$ trong những tình huống đặc biệt (hướng dẫn thực hiện các khiếu nại, đề xuất...).

Như vậy, kỹ năng đặt câu hỏi của CVHT có thể được định nghĩa là khả năng vận dụng kiến thức, kinh nghiệm của CVHT để chủ động khám phá, gợi mở vấn đề của $\mathrm{SV}$ một cách rõ ràng, với thái độ khích lệ, giúp SV nâng cao nhận thức và tự giải quyết vấn đề của mình trong học tập theo tín chỉ.

Mục đích của kỹ năng đặt câu hỏi trong hoạt động tư vấn của CVHT là tạo ra sự tương tác, giao tiếp tích cực giữa CVHT và $\mathrm{SV}$; Thu thập thông tin về những vấn đề, những khó khăn mà SV gặp phải; Khơi gợi tiềm năng của $\mathrm{SV}$ đối với vấn đề đang gặp; Giúp $\mathrm{SV}$ nhận thức được về bản thân và hoàn cảnh để có cách giải quyết hợp lý cho vấn đề đang gặp.

Yêu cầu đối với kỹ năng đặt câu hỏi: Đặt câu hỏi cần rõ ràng, mạch lạc, không hỏi nhiều ý; Xác định rõ mục tiêu khi hỏi; Để ý về thời điểm, tần suất các câu hỏi; Hỏi về cảm xúc, suy nghĩ của SV; Có phản hồi giữa những lần hỏi; Xử lý khéo léo trong những tình huống SV không muốn trả lời.

Biểu hiện kỹ năng đặt câu hỏi của CVHT được xác định qua các ý sau:

Biết cách đặt câu hỏi: Sử dụng câu hỏi hợp lý, linh hoạt, có nhiều câu hỏi mở; Câu hỏi bắt đầu với các từ như: cái gì? điều gì? kết thúc câu hỏi với các từ như: thế nào? ra sao; Sử dụng câu hỏi đóng một cách hạn chế, có suy xét (chỉ trong những trường hợp cần thiết).

Định hướng rõ nội dung hỏi: Hỏi về cảm xúc, suy nghĩ, hành vi của SV; Hỏi về thông tin liên quan hiện tại, không chỉ về quá khứ; Hỏi về suy nghĩ, mong muốn, nhu cầu và hướng giải pháp của chính $\mathrm{SV}$.

Làm chủ tiến trình hỏi: Xác định thời điểm, tần suất câu hỏi phù hợp, không dẫn dắt $\mathrm{SV}$ theo ý kiến chủ quan của mình, hỏi và chấp nhận cách $\mathrm{SV}$ trả lời nhanh hoặc chậm, không hối thúc, thể hiện thái độ khích lệ trong khi hỏi, tôn trọng, không phê phán, chấp nhận $\mathrm{SV}$, tôn trọng sự im lặng, dành thời gian cho SV suy nghĩ, có hành vi khích lệ như phản hồi, tóm lược, khen ngợi sau đó mới hỏi tiếp.

\subsubsection{Kỹ năng cung cấp thông tin}

Các nghiên cứu về hoạt động của CVHT đều cho rằng, hoạt động chủ đạo trong tư vấn của $\mathrm{CVHT}$ là cung cấp thông tin cho SV. Kỹ năng tư vấn là việc trao đổi, cung cấp thông tin và kết thúc thường là bằng hành động nào đó. Có thể mô tả như sau: CVHT sử dụng kỹ năng tư vấn "Để tiếp nhận thông tin của $S V$; CVHT phản hồi lại thông tin của $\mathrm{SV}$ (bằng cách tóm lược)" sau đó, CVHT cung cấp thông tin cho SV. Để cung cấp được thông tin tốt cho $\mathrm{SV}, \mathrm{CVHT}$ cần tích cực tìm kiếm, tích lũy tất cả các nguồn tin, còn trong 
quá trình cung cấp thông tin, CVHT cần lựa chọn thông tin khách quan, trung thực, phù hợp với đặc điểm của $\mathrm{SV}$ mà $\mathrm{CVHT}$ đang tư vấn. Mục đích cung cấp thông tin là chia sẻ trực tiếp về những sự thực, ý tưởng, giá trị và niềm tin của người tư vấn, do đó, điều quan trọng là người tư vấn cần có khả năng trong việc truyền đạt và có thái độ đúng đắn khi nói về bất kỳ thông tin nào mà họ có, vì những thông tin nhận được sẽ tác động rất lớn đến sự hiểu biết và sự trưởng thành của đối tượng, việc cung cấp thông tin cho các đối tượng cần căn cứ vào sự hiểu biết cẩn thận về nhu cầu của các đối tượng và cân nhắc tính hiệu quả đối với họ.

Như vậy, kỹ năng cung cấp thông tin của CVHT là khả năng vận dụng kiến thức, kinh nghiệm của người làm $\mathrm{CVHT}$ vào truyền đạt cho $\mathrm{SV}$ những thông tin có chất lượng, chính xác với thái độ tôn trọng, giúp SV nâng cao nhận thức và tự giải quyết vấn đề trong quá trình học tập theo tín chỉ.

Mục đích kỹ năng cung cấp thông tin trong hoạt động tư vấn của CVHT: Cung cấp cho SV biết những thông tin của các cấp (Ban Giám hiệu; các Phòng ban; Khoa...; cơ sở tuyển dụng; cơ sở thực tập...) để SV có thêm thông tin khách quan, chính xác, giúp các em nhìn nhận lại vấn đề và tự giải đáp thắc mắc, chọn lựa cách giải quyết cho vấn đề đang gặp phải từ thông tin được cung cấp, từ đó $\mathrm{SV}$ trưởng thành hơn trong đời sống nhờ các thông tin hữu ích mà CVHT mang lại.

Để cung cấp được thông tin tốt cho $\mathrm{SV}, \mathrm{CVHT}$ cần đáp ứng được yêu cầu: thu thập được những thông tin từ các nguồn thông tin đáng tin cậy, chính xác và khách quan, giúp SV nhìn nhận được toàn diện vấn đề, có kỹ năng truyền đạt thông tin rõ ràng, mạch lạc (tránh việc $\mathrm{SV}$ hiểu nhầm) và kiểm soát được cảm xúc của mình trong quá trình cung cấp thông tin (không nghiêm trọng hóa, hoặc đơn giản hóa vấn đề) để SV hình dung được tổng thể sự việc và lựa chọn được phương án giải quyết phù hợp.

Biểu hiện ở kỹ năng thu thập thông tin của CVHT được thể hiện qua các ý:

Nguồn thông tin chất lượng, khách quan: CVHT sử dụng các nguồn thông tin có cơ sở, chính xác, thiết thực, phù hợp với từng khóa học, năm học, giới tính, thông tin từ các Phòng, ban, Khoa, thông báo chính thức của nhà trường; từ các nhà chuyên môn, công trình nghiên cứu khoa học, sản phẩm ứng dụng, thông tin từ đơn vị có thẩm quyền, đại diện cho cơ quan truyền thông, cơ quan tuyển dụng,...

Cách thức truyền đạt thông tin: CVHT tóm tắt lại vấn đề $\mathrm{SV}$ cần tư vấn, khi cần thì ghi ra giấy các thông tin cho SV dễ kiểm tra lại, diễn đạt đúng, đủ, cụ thể về thông tin cần cung cấp, hỏi lại $\mathrm{SV}$ để kiểm chứng xem SV đã hiểu ý mình vừa trao đổi chưa, có thể hoạt động tư vấn được thực hiện gián tiếp qua điện thoại, thư điện tử [1, tr. 47-48].

Thái độ khi cung cấp thông tin (giọng nói, ánh mắt...): Thái độ khi trình bày thông tin cần thư giãn, không căng thẳng, không nhăn trán, nhíu mày gây khó hiểu cho $\mathrm{SV}$, mạnh dạn bày tỏ sự hạn chế của cá nhân trong việc cung cấp/trình bày thông tin để SV tự lựa chọn thông tin cần thiết, không can thiệp vào quyết định của các em.

\subsubsection{Kỹ năng khuyến khich, động viên}

Hoạt động tư vấn đều cần đưa đến giải pháp, nhưng điều quan trọng là $\mathrm{SV}$ cảm thấy vững vàng khi lựa chọn giải pháp và quyết tâm thực hiện. Điều này đòi hỏi CVHT phải thấu hiểu được tâm lý của $\mathrm{SV}$ và cần có kỹ năng động viên, khích lệ. Như vậy, một trong số các kỹ năng quan trọng của CVHT chính là động viên, khích lệ SV thực hiện giải pháp mà các em vừa lựa chọn, thông qua việc bày tỏ sự cảm thông, tán thành với $\mathrm{SV}$ về khúc mắc của các em; công nhận những điều tốt đẹp mà $\mathrm{SV}$ đã làm được, động viên các em thực hiện những quyết định mới.

Từ những ý kiến về kỹ năng động viên, khích lệ và từ thực tiễn công việc tư vấn của CVHT, chúng ta có thể hiểu kỹ năng động viên, khích lệ của CVHT là khả năng vận dụng kiến thức, kinh nghiệm của CVHT vào việc bày tỏ sự thấu hiểu vấn đề gặp phải của $\mathrm{SV}$, khuyến khích và hỗ trợ $\mathrm{SV}$ tự tin vào bản thân, giúp các em nâng cao nhận thức và năng lực tự giải quyết vấn đề của mình trong quá trình học tập theo tín chỉ.

Mục đích kỹ năng động viên, khích lệ của CVHT đối với $\mathrm{SV}$ thể hiện sự tin tưởng vào $\mathrm{SV}$, khen ngợi những việc $\mathrm{SV}$ đã thực hiện mà mình biết, tán thành với những suy nghĩ đúng đắn của $\mathrm{SV}$, giúp các em cảm thấy có động lực để thực hiện điều mong muốn, thể hiện sự sã̃n sàng trợ giúp khi SV cần.

Biểu hiện kỹ năng động viên, khích lệ của CVHT được thể hiện như sau: Bày tỏ sự thấu hiểu vấn đề của SV bằng cử chỉ (không lời): Gật đầu thể hiện sự chia sẻ, mắt mở hơi to lên, hai bàn tay mở ra khi nói chuyện, phản hồi bằng các từ ngắn: "ừ", "à", ánh mắt thông cảm hướng về phía $\mathrm{SV}$; Bày tỏ sự thấu hiểu với SV bằng lời nói: Lời nói thể hiện sự chấp nhận $\mathrm{SV}$, ủng hộ $\mathrm{SV}$, nói về việc giữ bí mật đối với những điều SV chia sẻ. 
Khuyến khích SV tự tin vào bản thân khi giải quyết các vấn đề của mình: Khen ngợi những gì $\mathrm{SV}$ đã hiểu tốt, làm đúng trong thời gian qua, thể hiện sự tin tưởng bằng ánh mắt, gật đầu để khuyến khích các em mạnh dạn xử lý các tình huống xảy ra, nói với SV: việc tìm đến CVHT ngầm ẩn sự nỗ lực của các em trong việc giải quyết vấn đề mà $\mathrm{SV}$ gặp phải.

Hỗ trợ giải quyết vấn đề: Gợi ý và phân tích cho SV thấy rõ lợi ích của từng giải pháp trong trường hợp của các em, hướng dẫn SV gặp ngay người có thể giải quyết việc này (vì kinh nghiệm từ trước đó đã có $\mathrm{SV}$ gặp vấn đề tương tự), ngỏ ý muốn tiếp tục hỗ trợ $\mathrm{SV}$ nếu các em chưa giải quyết được vấn đề của mình.

Từ những phân tích trên cho thấy, kỹ năng tư vấn của CVHT là tổ hợp của các kỹ năng thành phần, trong đó biểu hiện rõ nhất ở 4 nhóm kỹ năng: kỹ năng lắng nghe, kỹ năng đặt câu hỏi, kỹ năng cung cấp thông tin và kỹ năng động viên, khích lệ. Do đó, người làm công tác $\mathrm{CVHT}$ cần phải nắm bắt và hoàn thiện các kỹ năng trên để đáp ứng và nâng cao hiệu quả, nhiệm vụ CVHT của mình.

\section{Kết luận}

Như vậy, từ các phân tích trên cho thấy, đặc trưng của đào tạo theo tín chỉ là trao lại sự chủ động trong học tập cho SV, nhưng các em gặp không ít khó khăn trong việc định hướng, vì vậy, nhiều trường đã thành lập đội ngũ CVHT để tư vấn cho SV chọn lựa được cách học, quy trình học tập. Công tác tư vấn học tập của $\mathrm{CVHT}$ đóng vai trò hết sức quan trọng trong quá trình đào tạo theo HTTC. Tuy nhiên, để công tác tư vấn học tập thực sự đạt hiệu quả thì cần có sự quan tâm đúng mức tới kỹ năng tư vấn học tập của CVHT. Đồng thời, để hoạt động tư vấn đạt hiệu quả cần tuân thủ các yêu cầu: việc tư vấn, trợ giúp SV phải được tiến hành công bằng, công khai và đặt mục tiêu, lợi ích của SV lên hàng đầu; nội dung tư vấn phải trung thực, chính xác, không trái pháp luật và các quy định, quy chế của nhà trường; hoạt động tư vấn phải được ghi chép cẩn thận tại các cuộc họp, biểu mẫu và bản kế hoạch công tác cá nhân của CVHT. Theo chúng tôi, để thực hiện tốt các yêu cầu trên đòi hỏi $\mathrm{CVHT}$ vừa có trình độ chuyên môn vững vàng, khả năng giao tiếp tốt, đồng thời phải có phẩm chất đạo đức để sẵn sàng tư vấn, giúp đỡ $\mathrm{SV}$ trong mọi hoàn cảnh.

\section{REFERENCES}

[1]. Tran Cong Su (2016), Support system for learning advisors on mobile devices, Journal of Science, Can Tho University, No. 47/2016.

[2]. Tran Thi Minh Duc (2012), Study advisor in universities, National University Publishing House, Hanoi.

[3]. Luong Tu Hanh (2015), Some solutions to improve the quality of training through academic advising at universities, Educational Magazine, special issue December 2015.

[4]. Tran Van Phuc, Nguyen Kim Chuyen (2016), Development of academic advisor models at Dong Thap University in the current period, Educational Magazine, special issue December 2016.

[5]. Educational Research Institute - Ho Chi Minh City University of Education (2014), Proceedings of national seminars: "The role of academic advisor in credit training at colleges and universities. study in Vietnam ”, Ho Chi Minh City, 2014.

\section{CONSULTING ACTIVITIES OF STUDYING CONSULTANTS FOR STUDENTS IN UNIVERSITIES}

\section{Article info}

Recieved:

22/5/2020

Accepted:

20/9/2020

Keywords:

studying consultants,

consulting activities,

students, lecturers,

universities.

\begin{abstract}
At universities in Vietnam, the credit-based training system has become a mandatory regulation of Ministry of Education and Training to support students to ferform their autonomy in the most effective way. An important part of the creditbased training procedure is the board of studying consultants who play the role of a homeroom teacher to manage students, and they also are studying consultants, scientific and career researchers for students. The article mentions concepts, content and skills that are necessary to consult students in universities.
\end{abstract}

